\title{
A Influência dos Estágios do Ciclo de Vida Organizacional no Uso de Medidas de Desempenho em Empresas Industriais
}

\section{The Influence of Organizational Life Cycle of Stages on the Use of Performance Measures in Industrial Companies}

\author{
Gilvan Medeiros de Santana Junior ${ }^{* 1}$, Aldo Leonardo Cunha Callado ${ }^{2}$ \\ ${ }^{1}$ Instituto Federal de Educação, Ciência e Tecnologia da Paraíba, Paraíba, Brasil. \\ ${ }^{2}$ Universidade Federal da Paraíba, Paraíba, Brasil.
}

\begin{tabular}{l} 
I N F O A R T I G O \\
\hline Palavras-chave: \\
Ciclo de Vida \\
Organizacional, \\
Mensuração de \\
Desempenho, \\
Medidas de Desempenho \\
Financeiras, \\
Medidas de Desempenho \\
não Financeiras, \\
Segmento Industrial.
\end{tabular}

\section{ARTICLE INFO}

\section{Keywords:}

Organizational Life Cycle, Performance Measurement, Measures of Financial Performance,

Non-Financial Performance Measures, Industrial Segment.

\begin{abstract}
RESUMO
Baseado na necessidade de desenvolver pesquisas no contexto brasileiro envolvendo ciclo de vida organizacional (CVO) e controles gerenciais (CUNHA; KLANN; LAVARDA, 2013), esta pesquisa analisou a influência do CVO no uso das medidas de desempenho em empresas industriais da Paraíba. Para tanto, utilizou-se do modelo de Lester, Parnell e Carraher (2003) para identificar os estágios do ciclo de vida que se encontravam as empresas industriais. E por meio de 39 medidas de desempenho, mensurouse o nível de intensidade de uso das medidas. A partir da análise discriminante e da análise de regressão, identificou-se que há influência dos estágios do CVO no uso das medidas de desempenho. Contudo, nem todos os estágios do CVO exerceram influências no uso das medidas de desempenho. Concluiu-se que as empresas usam em menor intensidade as medidas de desempenho nos casos em que elas apresentem características do estágio nascimento, contradizendo a literatura que afirma que nos estágios iniciais do ciclo vida as empresas dão preferências ao uso de medidas de desempenho não financeiras.
\end{abstract}

\section{A B S T R A C T}

Based on the lack of national studies involving organizational life cycle (CVO) and management controls (CUNHA, KLANN, LAVARDA, 2013), this study analyzed the influence of $\mathrm{CVO}$ on the use of performance measures in industrial companies in Paraíba. For this purpose, the model of Lester, Parnell and Carraher (2003) was used to identify the stages of the life cycle that were the industrial companies. And by means of 39 measures of performance, the level of intensity of use of the measures was measured. From the discriminant analysis and the regression analysis, it was identified that there is influence of the stages of the CVO in the use of the performance measures. However, not all stages of the CVO have influenced in the use of performance measures. It was concluded that companies use performance measures to a lesser extent in cases where they present characteristics of the birth stage, contradicting the literature that states that in the early stages of life, companies give preference to the use of non-financial performance measures.

\footnotetext{
* Correspondência para autor: gilvan.santana@ifpb.edu.br (Santana Junior, G. M. de), aldocallado@yahoo.com.br (Callado, A. L. C.).
} 


\section{Introdução}

Estudos apontam que as organizações passam por diversas fases à medida que crescem e se desenvolvem, formando assim um ciclo de vida organizacional (HANKS et al., 1993; MILLER; FRIESEN, 1984; MINTZBERG, 1984). À medida que essas empresas se deslocam entre os estágios do ciclo de vida, diferentes oportunidades e ameaças exercem pressões para que as empresas se adaptem a novos cenários, exigindo da alta gestão, alterações nos objetivos, nas estratégias da empresa, nos processos gerenciais, no estilo de tomada de decisões, entre outros.

A literatura associada ao ciclo de vida organizacional $(\mathrm{CVO})$ indica que há várias razões pelas quais o uso de controles gerenciais, incluindo medidas de desempenho, difere entre os diversos estágios de vida organizacional. Estas razões são em virtude das mudanças organizacionais que provocam alterações nas atividades administrativas, nos ambientes de negócios, nas estratégias e nas estruturas de organizações entre as empresas em diferentes fases do ciclo de vida. Dessa forma, a avaliação de desempenho, considerando os diferentes cenários em que a empresa esteja inserida, exige que diferentes medidas de desempenho sejam usadas (KALLUNKI; SILVOLA, 2008).

No tocante aos processos gerenciais que são influenciados pelo ciclo de vida organizacional, Garengo, Nudurupati e Bititci (2007) afirmam que organizações em estágios de vida mais avançados são mais capazes de reconhecer suas necessidades organizacionais, e consequentemente, projetar o sistema de informação gerencial de modo que atenda as necessidades de mensuração de desempenho. Assim, concluem que os estágios do ciclo de vida organizacional são fatores essenciais que interferem na medição de desempenho organizacional.

As medidas de desempenho são importantes instrumentos de gestão, pois através delas, os gestores podem monitorar e avaliar os resultados organizacionais, bem como diagnosticar e corrigir os desvios de padrões pré-estabelecidos de desempenho. Dessa forma, os indicadores de desempenho, além de ajudar os gestores a alcançar os objetivos da organização, também os auxiliam na melhor aplicação dos recursos, e no aperfeiçoamento o desempenho organizacional, tornando-a mais competitiva (SIMONS, 1995; CHAVES; ALCÂNTARA; ASSUMPÇAO, 2008).

A proposta desse estudo surgiu a partir da identificação de pesquisas que investigaram a existência de associação entre os estágios do ciclo de vida organizacional (CVO) a as práticas de controles gerenciais, a exemplo de planejamento estratégico; orçamentos, sistemas de custeio, dentre outras. Dentre as diversas práticas gerenciais investigadas, não se identificou um estudo que tenha verificado a relação entre os estágios do CVO e o uso de medidas de desempenho (financeiras e não financeiras).

Tal ausência, percebida em estudos que analisam os controles gerenciais nos diferentes estágios do ciclo de vida organizacional, sobretudo quanto ao uso de medidas de desempenho, é objeto deste estudo e foi indicada por Cunha, Klann e Lavarda (2013). Sendo assim, a presente pesquisa buscou responder a seguinte questão: qual a influência dos estágios do ciclo de vida organizacional no uso de medidas de desempenho (financeiras e não financeiras) em empresas industriais da Paraíba?

Vários são os modelos de ciclo de vida organizacional identificados na literatura (GREINER, 1972; CHURCHILL; LEWIS, 1983; MILLER; FRIESEN, 1984; SCOTT; BRUCE, 1987; ADIZES, 1990), cuja principal variável que os diferenciam é a quantidade de estágios, uns considerados mais gerais, por terem um número mais reduzido de etapas, e outros específicos, por compreenderem mais etapas. Correia et al. (2010) verificaram, na literatura internacional, uma predominância na utilização do modelo proposto por Miller e Friesen (1984).

Porém, o referido modelo apresenta algumas limitações, a exemplo de não poder ser aplicado em qualquer tipo de organização. Outro aspecto apontado pelo estudo é que o modelo desenvolvido por Lester, Parnell e Carraher (2003) vem se destacando dentre os demais por apresentar uma escala estatisticamente validada que pode ser aplicada em qualquer tipo de organização. Diante do exposto, para fins deste estudo, utilizou-se o modelo proposto por Lester, Parnell e Carraher (2003) pela possibilidade de ser aplicado em qualquer organização. 
Além desta introdução, o trabalho está dividido em 4 seções. Na seção 2 é realizada uma revisão da literatura, abordando o Ciclo de Vida Organizacional (CVO), a Mensuração de Desempenho e os estudos já desenvolvidos e que são relacionados ao tema. $\mathrm{Na}$ seção seguinte, apresentam-se os procedimentos metodológicos que foram adotados para a realização da pesquisa. Na seção subsequente, são apresentados os resultados encontrados. E por fim, as considerações finais.

\section{Referencial Teórico}

Nesta seção são abordados os principais aspectos conceituais da Teoria do Ciclo de Vida Organizacional e da Mensuração de Desempenho. Na abordagem teórica do ciclo de vida organizacional, apresenta-se o modelo proposto por Lester, Parnell e Carraher (2003) que foi utilizado como parâmetro para classificar as empresas participantes desta pesquisa nos respectivos estágios do ciclo de vida. Em seguida, apresentam-se conceitos, objetivos e importância da mensuração de desempenho. Por fim, são apresentados os estudos anteriores desenvolvidos que trataram sobre o ciclo de vida organizacional.

\subsection{O Ciclo de Vida Organizacional}

A Teoria do Ciclo de Vida Organizacional (CVO) associa o processo evolutivo das organizações aos dos seres humanos, que, por meio da adaptação de conceitos das ciências biológicas, apresentam que as organizações nascem, crescem de diferentes formas e morrem (LESTER; PARNELL; CARRAHER, 2003; MINTZBERG, 1984; MILLER; FRIESEN, 1984; QUINN; CAMERON, 1983). No entanto, é possível verificar na literatura que as empresas não seguem uma ordem cronológica, necessariamente, assim como acontece com a vida dos seres humanos (LESTER; PARNELL; CARRAHER, 2003; SANTOS; CORREAA; BEUREN, 2016).

Nesse contexto, é possível que as organizações voltem a um estágio anterior ou permaneçam em um estágio de desenvolvimento por um longo período de tempo, tendo em vista que, tamanho e idade da organização não são fatores determinantes no crescimento e envelhecimento (BORINELLI, 1998). Portanto, na análise do ciclo de vida organizacional, tamanho e tempo de fundação não são causas de crescimento e envelhecimento.

Sendo assim, os estágios de CVO representam um conjunto de atividades organizacionais e estruturais que pode ser identificado por meio de uma interpretação do meio ambiente em que a organização se encontra e com base em uma avaliação desenvolvida com os gestores. Para Hanks et al. (1993), as fases de ciclo de vida organizacional são definidas por meio de uma configuração única de variáveis relacionadas ao contexto, estratégia e estrutura organizacional.

Para Lester, Parnell e Carraher (2003), o conhecimento do estágio de vida organizacional, em que as firmas se encontram, tem sua importância elevada à medida que proporciona aos gestores uma melhor compreensão das relações entre o ciclo de vida organizacional, estratégia competitiva e desempenho. Os mesmos autores investigaram o desenvolvimento do ciclo de vida organizacional e propuseram e testaram empiricamente um modelo que contempla cinco estágios, a fim de avaliar as fases específicas do ciclo de vida de qualquer organização.

O modelo de cinco estágios proposto por Lester, Parnell e Carraher (2003) foi desenvolvido a partir dos atributos reunidos em vários outros estudos, como os de Adizes (1990), Flamholtz (1986) e Miller e Friesen (1984). Então, por meio de uma escala de vinte itens, Lester, Parnell e Carraher (2003) captaram a percepção dos gestores com relação à posição de empresas associadas às seguintesfases: nascimento, crescimento, maturidade, rejuvenescimento e declínio.

O primeiro estágio, nascimento, é marcado pelo início do desenvolvimento organizacional. As principais características dessa fase são: decisão centralizada nas mãos de um indivíduo, geralmente, o proprietário; e preocupação em identificar um número suficiente de clientes para apoiar a existência da organização.

A busca por geração de receitas suficientes para continuar as operações e para se manter competitiva é o principal objetivo do estágio crescimento. Nesta fase, desenvolve-se alguma formalização de estrutura e estabelecem-se as suas próprias competências distintivas.

Maturidade é o estágio marcado pelo alto nível de formalidade e controles organizacionais, cujas descrições de trabalho, políticas, procedimentos e relações de subordinação hierárquica se tornam muito mais 
formais.

Rejuvenescimento, o quarto estágio do modelo de ciclo de vida organizacional proposto por Lester, Parnell e Carraher (2003), centra-se nos processos de inovação e criatividade, e por apresentar um processo de tomada de decisão bastante descentralizado. Apesar de a estrutura burocrática fazer parte desse estágio organizacional, os colaboradores são incentivados a trabalharem dentro da burocracia, sem que tenham de acrescentar algo, isto é, os trabalhadores são incentivados a trabalharem diante dos processos já formalizados.

Por fim, o último estágio, o declínio que segundo Lester, Parnell e Carraher (2003) é marcado pela política, poder e pelos membros das organizações tornarem-se mais preocupados com a realização de seus objetivos pessoais, em detrimento das metas organizacionais.

Portanto, diante do exposto, e considerando o estudo de Correia et al. (2010), que apontou que o modelo de cinco estágios desenvolvido por Lester, Parnell e Carraher (2003) se destaca por apresentar uma escala estatisticamente validada e que pode ser aplicada em qualquer tipo de organização, este trabalho fez uso desse modelo para classificar as empresas nos diferentes estágios de vida.

A seguir, serão abordados os principais conceitos da mensuração de desempenho, tendo em vista que este estudo tem o objetivo de analisar a influência dos estágios de vida no uso de medidas de desempenho.

\subsection{Mensuração de Desempenho}

A mensuração de desempenho tem sido um tema bastante discutido na literatura internacional e nacional, em razão de ser um importante instrumento de monitoramento e controle à gestão empresarial. Também é considerada uma ferramenta relevante para influenciar o comportamento e ações dos colaboradores, uma vez que, possibilita verificar a eficiência e eficácia de ações de um grupo de indivíduos.

Para Neely, Gregory e Platts (2005), a mensuração de desempenho pode ser definida como o processo que, por meio de medidas de desempenho, quantifica a eficiência e eficácia de uma ação. Os autores esclarecem que as medidas de desempenho consistem em uma métrica usada para quantificar a eficiência e/ou a eficácia de uma ação.

O objetivo da medição de desempenho está associado à visão da organização. Sendo assim, há várias formas e medidas para avaliar o desempenho das empresas. No entanto, uma das preocupações da mensuração de desempenho é definir a lista de indicadores que será utilizada, visto que as métricas precisam estar alinhadas com as metas organizacionais (RIBEIRO; MACEDO; MARQUES, 2012; CALLADO, 2016).

Nessa perspectiva, estudos apontam que o contexto empresarial, traduzido por meio da teoria do ciclo de vida organizacional, é um dos fatores determinantes que interferem na escolha de quais medidas de desempenho devem ser adotadas para avaliar o desempenho organizacional (YAN; GRAY, 1995; LARIMO; NGUYEN; ALI, 2016).

A relevância das medidas de desempenho é destacada por Bond (2002) ao afirmar que a formulação de estratégias, o desenvolvimento de ações, programas e processos de melhoria e mudanças das operações passam, necessariamente, pela criação de métricas que monitoram seus estágios passados e atuais, permitindo previsões para o futuro.

Os benefícios gerados pelo processo de avaliação são: redução de custos, melhorias na qualidade dos serviços, aumento do nível de satisfação dos clientes o que pode acarretar em incremento das receitas (NEELY, 2002). De acordo com Konsta e Plorimatou (2012), a entidade que não consegue avaliar seu desempenho, não consegue acompanhar suas atividades, e consequentemente, não consegue implantar medidas essenciais que tenham a finalidade de melhorar a situação econômico-financeira.

As medidas de desempenho possuem diversas classificações, podendo ser inicialmente relatadas em termos financeiros ou não financeiros (operacionais). Além destas, é possível encontrar uma terceira classificação que é a combinação de medidas financeiras e não financeiras (MIRANDA et al., 2003; LOPES; BEUREN, 2015; VARGAS et al., 2016).

Entende-se que medidas financeiras são indicadores expressos em valores provenientes da contabilidade financeira, obtidas por meio dos relatórios contábeis. Contudo, os indicadores não financeiros consistem em métricas que procuram alinhar o desempenho da companhia com os seus objetivos organizacionais (CALLADO; CALLADO; WEGNER, 2015). 
O surgimento das medidas de desempenho não financeiras se deu como sendo um instrumento capaz de auxiliar a tomada de decisão em um ambiente em que as informações financeiras (tradicionais) e contábeis já não eram suficientes para mensurar o desempenho da organização (LOPES; BEUREN, 2015).

Portanto, nota-se que a mensuração de desempenho exerce um importante papel no processo gerencial das empresas e que ao longo do tempo houve um desenvolvimento nos tipos de medidas de desempenho, passando mais especificamente a ter duas classificações: medidas financeiras e medidas não financeiras. $\mathrm{O}$ surgimento das medidas não financeiras se deu, em razão da incapacidade das medidas financeiras em avaliar com eficiência e eficácia as ações empresariais em tempo real.

\subsection{Estudos Anteriores}

Diversos trabalhos, tanto na literatura internacional, quanto na literatura nacional, analisaram a associação da teoria do ciclo de vida com os controles gerenciais das empresas (MOORES; YUEN, 2001; LESTER; PARNELL; CARRAHER, 2003; AUZAIR; LANGFIELD-SMITH, 2005; GRANLUND; TAIPALEENMAKI, 2005; GARENGO; NUDURUPATI; BITITCI, 2007; KALLUNKI; SILVOLA, 2008; SOUZA; FREZATTI; NECYK, 2008; NECYK; FREZATTI, 2010; FREZATTI et al., 2010; BEUREN; RENGEL; HEIN, 2012; BEUREN; RENGEL; RODRIGUES JUNIOR, 2015; SANTOS; CORREA; BEUREN, 2016; FREZATTI et al., 2017; ALMEIDA; WERNKE, 2018; SILVA; MARQUES; CECON, 2020).

Moores e Yuen (2001) examinaram a natureza de diferentes níveis de formalidade do Sistema de Informação de Contabilidade Gerencial no que se refere à natureza da seleção e apresentação da informação contábil gerencial ao longo do ciclo de vida das empresas (nascimento, crescimento, maturidade, rejuvenescimento e declínio). Segundo os autores, as empresas no estágio da maturidade tendem a estabilizar suas operações e aumentam a quantidade de informações para a tomada de decisão. Os autores identificaram que a fase de rejuvenescimento é marcada pela utilização de estratégias de diversificação e expansão de produtos, momento em que as empresas fazem uso de uma gama de instrumentos gerenciais para fornecimento de informações em maior proporção do que as empresas do estágio de crescimento e maturidade.

Kallunki e Silvola (2008) investigaram se o uso de sistemas de contabilidade de custo baseado em atividades difere entre os estágios do ciclo de vida organizacional. A partir de uma amostra composta por 105 empresas, os autores concluíram que o uso dos sistemas de contabilidade de custo é afetado pelos diversos estágios do ciclo de vida organizacional, observando que as empresas em fases de maturidade e rejuvenescimento apresentam um maior uso do sistema em relação às empresas que estavam na fase de crescimento. Outra conclusão apontada pelos autores foi que o estágio de ciclo de vida é determinante para explicar a utilização do custeio baseado em atividades entre empresas, ao invés do tamanho ou idade da empresa.

Souza, Frezatti e Necyk (2008) realizaram um ensaio teórico com o objetivo de ampliar o conhecimento de como a contabilidade gerencial se desenvolve ao longo do tempo dentro de uma organização, tomando como base o modelo de ciclo de vida. Os autores demonstraram o potencial que a abordagem do ciclo de vida oferece no desenvolvimento do entendimento da contabilidade gerencial, ao comprovar que características da contabilidade gerencial sofrem influências dos estágios do ciclo de vida organizacional.

Frezatti et al. (2010) desenvolveram uma pesquisa em que analisaram o perfil das empresas brasileiras, associando a estruturação do processo de planejamento aos estágios do ciclo de vida organizacional. Os autores constataram haver correlação entre os estágios do ciclo de vida organizacional e o perfil de fomalização do planejamento nas empresas. No entanto, observaram a não existência do intrumento gerencial, orçamento, nos estágios associados ao nascimento e declínio, destacando, nesse último estágio, que o orçamento deveria ser entendido como uma alavanca de recuperação.

Santos, Corrêa e Beuren (2016) utilizaram o modelo de Lester, Parnell e Caraher (2003) para estudar a relação entre ciclo de vida organizacional e o uso de instrumentos tradicionais e modernos de contabilidade gerencial. Em seus resultados, os autores identificaram que a maioria das empresas encontra-se no estágio nascimento, com diferentes anos de constituição, não seguindo uma ordem determinística. Constataram relações significantes positivas e negativas entre os estágios de ciclo de vida e os instrumentos gerenciais. No entanto, apenas os métodos de custeio (absorção, variável e padrão) não apresentaram relação significativa com os estágios do ciclo de vida. 
Frezatti et al. (2017), por meio de uma amostra de 117 empresas familiares e se utilizando do modelo de CVO de Lester, Parnell, Carraher (2003), analisaram a relação entre os estágios do ciclo de vida organizacional e os elementos de influência da família na empresa no modelo F-PEC (Poder, Experiência e Cultura). Como resultado, identificaram que no nascimento, o poder e a cultura têm papéis importantes; na maturidade, a experiência e a cultura se destacam; e, no rejuvenescimento, o poder e a cultura foram identificados como caracterizadores.

Almeida e Wernke (2018) investigaram os estilos gerenciais dos dirigentes de pequenas empresas baseado no ciclo de vida organizacional. Por meio de uma amostra de 45 pequenas empresas, os autores investigaram quatro grupos de estilos (orientação, direção, relações públicas e solucionador de distúrbios). A principal contribuição do estudo residiu na geração de subsídios para que as instituições de ensino superior e entidades empresariais da região elaborem políticas de formação de dirigentes de forma mais customizada, contemplando gestores com formações em diferentes áreas. Cabe destacar que os autores apontam como limitação do estudo, o tamanho da amostra utilizada.

Silva, Marques e Cecon (2020) a partir de uma amostra de 44 empresas listadas na bolsa de valores do Brasil (B3), investigaram a relação entre os artefatos de contabilidade gerencial empregados pelas empresas do setor de consumo cíclico e os estágios de ciclo de vida. Como resultado, o estudo identificou que as empresas no estágio de crescimento tendem a utilizar artefatos de contabilidade gerencial caracterizados pela literatura como modernos, e as empresas no estágio de maturidade tendem a utilizar artefatos caracterizados como tradicionais. Por sua vez, o estágio de declínio não apresentou proximidade com nenhuma das categorias de artefatos.

Sendo assim, embora haja trabalhos que tenham analisado a associação entre os estágios do ciclo de vida organizacional (CVO) e diversos tipos de controles gerenciais, este trabalho se diferencia ao estudar a relação entre dois temas pouco explorado conjuntamente que são: Ciclo de Vida Organizacional e Mensuração de Desempenho.

\section{Metodologia}

O presente estudo possui características de natureza descritiva com abordagem quantitativa. De acordo com Gil (2010), esse tipo de pesquisa tem como objetivo principal a descrição das características de determinada população ou fenômeno ou o estabelecimento de relação entre variáveis. O presente estudo analisou a relação entre duas variáveis: ciclo de vida organizacional e medidas de desempenho organizacional. Para Richardson (1999), a pesquisa quantitativa “[...] caracteriza-se pelo emprego da quantificação tanto nas modalidades de coleta de informações quanto no tratamento desses dados através de técnicas estatísticas desde as mais simples às mais complexas, como, coeficiente de correlação, análise de regressão, etc.”.

\subsection{Hipótese da Pequisa}

Marconi e Lakatos (2015) reconhecem que, no início de qualquer investigação científica, devem-se formular hipóteses e que sua utilização é necessária para que a pesquisa apresente resultados úteis, ou seja, atinja níveis mais altos de interpretação.

Podem-se encontrar diversas definições para o termo hipótese. No entanto, Marconi e Lakatos (2015) a definem como sendo uma suposição que antecede a constatação dos fatos e tem como característica, uma formulação provisória que deve ser testada para determinar sua validade. Gil (2010) apresenta vários tipos de hipóteses: as causuísticas; as que se referem à frequência de acontecimentos; e as que estabelecem relações entre variáveis. Esta pesquisa se enquadra no terceito tipo, por analisar a influência de variáveis que são: ciclo de vida organizacional e medidas de desempenho organizacional.

Assim, considerando que os objetivos empresariais sofrem mudanças à medida que o contexto organizacional se altera, causando com isso, uma necessidade de avaliação de desempenho diferente, os gestores devem escolher medidas de desempenho diferentes, podendo variar no uso mais intenso de medidas financeiras ou não financeiras, a depender da fase de vida em que a organização se encontre.

As teorias que explicam as configurações organizacionais, a exemplo da teoria do ciclo de vida organizacional, afirmam que as mudanças empresariais afetam os controles gerenciais, inclusive, as medidas de desempenho. Assim, considerando que as medidas de desempenho têm a finalidade de medir se os 
objetivos estão sendo alcançados em um determinado período e como os objetivos são alterados na medida em que a empresa passa de um estágio de vida para outro, espera-se que elas também sejam modificadas.

Segundo Geringer e Hebert (1991), o uso de medidas financeiras tradicionais é mais adequado e frequentemente observado em organizações que se encontram em estágios de vida mais avançados. Em consonância com esses autores, Larimo, Nguyen e Ali (2016) afirmam que, para avaliar o desempenho organizacional, as empresas têm certas preferências por usar medidas não financeiras em estágios de formação, ou seja, nos estágios iniciais de vida e medidas financeiras em momentos de pós-formação. Assim, por meio do método hipotético-dedutivo, o presente estudo apresenta como hipótese de pesquisa a seguinte proposição:

H0: Os estágios iniciais (nascimento e crescimento) exercerão influência positiva nas medidas de desempenho não financeiras maiores do que nas medidas financeiras, quando comparados com os demais estágios.

\subsection{Universo e Amostra da pesquisa}

O universo da pesquisa compreendeu 3.321 empresas industriais localizadas no Estado da Paraíba e cadastradas na Federação das Indústrias do Estado da Paraíba - FIEP/PB. Foram excluídas 1.129 empresas por apresentar falta de informações no cadastro, a exemplo de endereços eletrônicos e telefones de contato.

A amostra, segundo Marconi e Lakatos (2015), pode ser definida como sendo "uma porção ou parcela, convenientemente selecionada do universo (população)". Quanto à amostragem, esse estudo é do tipo não probabilístico por acessibilidade ou por conveniência. Segundo Gil (2010), nesse tipo de amostragem, "o pesquisador seleciona os elementos a que tem acesso, admitindo que estes possam de alguma forma representar o universo".

Sendo assim, a amostra foi constituída pelos retornos obtidos por meio do envio do instrumento de coleta de dados, que ocorreu entre os meses de agosto e dezembro de 2016, e contou com a participação de 50 empresas industriais do Estado da Paraíba.

A amostra da pesquisa é representada por 14 setores econômicos, dos quais se destacam os setores da "Fabricação de Produtos Alimentícios" com participação de 34\% do total da amostra. Em seguida, aparecem "Confecção de Artigos do Vestuário e Acessórios" (14\%) e "Fabricação de Produtos Têxteis" e "Fabricação de Produtos de Minerais Não metálicos", com 8\% cada. Esses 4 setores juntos correspondem a $64 \%$ da amostra.

O restante 36\% estão distribuídos em 10 setores econômicos, sendo eles: "Fabricação de Bebidas" e "Construção de Edifícios" (6\% cada); "Impressão e Reprodução de Gravações", "Fabricação de Produtos Químicos", "Fabricação de Produtos de Metal, Exceto Máquinas e Equipamentos e "Fabricação de Produtos Diversos" (4\% cada); "Extração de Minerais Não-Metálicos", "Fabricação de Produtos de Borracha e de Material Plástico", "Metalurgia" e "Obras de Infraestrutura" (2\% cada).

Quanto ao perfil dos participantes, constata-se uma amostra com alto grau de escolaridade, tendo em vista que a maioria dos respondentes $(76 \%)$ concluiu o nível superior ou cursou uma pós-graduação. Verificou-se também que os profissionais possuem um bom tempo de experiência, já que mais de $60 \%$ afirmaram ter mais de 5 anos na função desempenhada atualmente, e mais de $76 \%$ possuem mais de 5 anos de experiência no segmento em que atua.

Nessa perspectiva, espera-se que os participantes possuam habilidades suficientes para responder aos quesitos, no que concerne ao contexto organizacional, estratégia e estrutura, variáveis (consideradas determinantes na definição das fases do ciclo de vida organizacional), bem como às questões referentes à utilização de medidas de desempenho.

\subsection{Instrumento de pesquisa e coleta de dados}

Para o desenvolvimento deste estudo foi utilizado o questionário, que, segundo Gil (2010), é uma técnica composta por um conjunto de questões submetidas a pessoas com o propósito de obter informações. Assim sendo, a coleta de dados se deu por meio do envio de um questionário estruturado do tipo fechado, formado por perguntas dicotômicas e de múltiplas escolhas, para o endereço eletrônico (e-mail) dos gestores das empresas. 
As variáveis relacionadas com os estágios do ciclo de vida foram traduzidas para o português do estudo de Lester, Parnell e Carraher (2003). E em seguida, comparadas e adaptadas com a pesquisa realizada por Beuren, Rengel e Rodrigues Junior (2015) que adotaram o mesmo modelo.

No caso das variáveis que verificavam o uso das medidas de mensuração de desempenho organizacional, extraiu-se do estudo de Callado, Callado e Almeida (2007), em razão de o trabalho ter sido realizado no contexto paraibano, ou seja, junto às empresas paraibanas. Os autores investigaram a utilização de indicadores de desempenho em agroindústrias. Ambas as variáveis, ciclo de vida organizacional e medidas de desempenho, sofreram adaptações com os ajustes provenientes da fase do pré-teste.

O pré-teste foi efetuado em duas etapas, com a finalidade de detectar possíveis falhas na redação do questionário, tais como: complexidade das questões, imprecisão na redação, desnecessidade de questões, exaustão e outros (GIL, 2010). Na primeira fase, o questionário foi enviado aos especialistas com experiências em pesquisas, sobretudo, associada ao tema ciclo de vida organizacional. E o segundo pré-teste foi realizado com dois gestores de empresas pertencentes ao universo deste estudo.

Após a realização dos pré-testes, as empresas foram convidardas, por meio de contato telefônico e via endereço eletrônico, a participar da pesquisa. Em seguida, os questionários foram enviados às empresas que concordaram em participar do estudo, anexando-se aos e-mails, uma carta explicando a natureza da pesquisa, sua importância e a necessidade em se obter resposta. Esse procedimento visou a despertar o interesse do receptor para o preenchimento e resposta do questionário dentro de um prazo razoável (MARCONI; LAKATOS, 2015).

\subsection{Variáveis da pesquisa}

As variáveis dependentes utilizadas neste estudo foi a média da intensidade de uso das medidas de desempenho (financeiras e não financeiras).

Nesse estudo, as medidas de desempenho utilizadas foram adaptadas a partir do estudo de Callado, Callado e Almeida (2007). As medidas foram submetidas à validação de especialistas e de empresas pertencentes ao universo, sendo necessárias algumas adaptações. As medidas utilizadas neste estudo estão apresentadas no Quadro 1.

Quadro 1 - Medidas de desempenho organizacional (financeira não financeira)

\begin{tabular}{|c|c|}
\hline $\begin{array}{c}\text { Medidas de desempenho } \\
\text { Financeira }\end{array}$ & $\begin{array}{c}\text { Medidas de desempenho } \\
\text { não financeira }\end{array}$ \\
\hline $\begin{array}{l}\text { Custo unitário do produto } \\
\text { Preço de venda estimado x realizado } \\
\text { Montante do resultado (Lucro ou Prejuízo) } \\
\text { Margem de lucro } \\
\text { Evolução do montante do faturamento (vendas) } \\
\text { Nível de inadimplência dos clientes } \\
\text { Nível de endividamento da empresa } \\
\text { Rentabilidade do patrimônio } \\
\text { Participação do produto no faturamento (vendas) } \\
\text { Participação do produto no resultado (Lucro ou Prejuízo) } \\
\text { Faturamento estimado x realizado } \\
\text { Margem de contribuição } \\
\text { Fluxo de caixa (entradas e saídas de caixa) } \\
\text { Evolução do montante dos investimentos } \\
\text { Rotação de estoque } \\
\text { Valor da empresa (preço de mercado) } \\
\text { Valor dos ativos (conjunto dos bens em geral) } \\
\text { Valor econômico agregado } \\
\text { Retorno sobre o investimento } \\
\text { Participação do representante/vendedor no faturamento } \\
\text { (vendas) } \\
\text { Valor do patrimônio }\end{array}$ & $\begin{array}{l}\text { Nível de qualidade do produto } \\
\text { Grau de participação da sua empresa no } \\
\text { mercado } \\
\text { Número de reclamações de clientes } \\
\text { Evolução do número de clientes } \\
\text { Grau de fidelidade dos clientes } \\
\text { Tempo de atendimento ao cliente } \\
\text { Evolução do volume de vendas } \\
\text { Grau de inovação } \\
\text { Rotatividade de empregados } \\
\text { Solução de reclamações } \\
\text { Número de funcionários } \\
\text { Perfil do consumidor (característica do cliente) } \\
\text { Capacidade de produção } \\
\text { Valor da marca }\end{array}$ \\
\hline
\end{tabular}


Nível de investimento em treinamento Nível de investimento em modernização Investimento em propaganda Investimento em ação social

Fonte: Adaptado de Callado, Callado e Almeida (2007).

Observa-se no Quadro 1 que as medidas de desempenho estão divididas em dois grupos: medidas de desempenho financeiras e medidas de desempenho não financeiras. Essa separação está de acordo com os estudos conduzidos por Miranda et al. (2003), Lopes e Beuren (2015) e Vargas et al. (2016) que mencionaram que as medidas de desempenho podem ser relatadas em termos financeiros ou não financeiros.

Ressalta-se que as medidas de desempenho utilizadas por Callado, Callado e Almeida (2007) foram selecionadas a partir da pesquisa conduzida por Miranda e Silva (2002). Estes autores, afirmam existir dois tipos de medidas: as absolutas e as relativas. Os dois tipos de medidas tanto podem ser classificadas como financeiras ou não financeiras. Sendo, as medidas absolutas expressas em números absolutos, como o valor do lucro (medida financeira) ou o volume de venda (medida não financeira). E as medidas relativas compreendem o resultado da comparação de dois indicadores, normalmente, expresso em forma percentual.

Um conjunto de variáveis independentes foi utilizado com a finalidade de analisar a influência do ciclo de vida organizacional (CVO) no uso das medidas de desempenho. Nesse caso, utilizaram-se as seguintes variáveis: (a) Estágios do ciclo de vida organizacional; (b) Tamanho da organização; (c) Diversidade de produtos; e $(d)$ Nível de escolaridade.

No que se refere a variável independente, estágios do ciclo de vida organizacional, utilizou-se o modelo de cinco estágios de CVO de Lester, Parnell e Carraher (2003). Os autores desenvolveram e testaram empiricamente um modelo de ciclo de vida em que, a partir de uma escala de 20 itens, avaliam as fases específicas do ciclo de vida de qualquer organização. O Quadro 2 apresenta os itens que caracterizam os estágios de vida do modelo de ciclo de vida utilizados nesta pesquisa.

Quadro 2 - Variáveis de identificação dos estágios do ciclo de vida organizacional

\begin{tabular}{|c|c|}
\hline Estágios & Variáveis de identificação de cada estágio do ciclo de vida \\
\hline \multirow{4}{*}{ 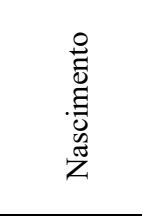 } & Nossa organização é pequena em tamanho, quando comparada com nossos concorrentes. \\
\hline & O poder decisório de nossa empresa está principalmente nas mãos do fundador. \\
\hline & A estrutura organizacional da nossa empresa pode ser considerada simples. \\
\hline & $\begin{array}{l}\text { O processamento de informação em nossa empresa pode ser descrito como simples, no estilo "boca- } \\
\text { a-boca". }\end{array}$ \\
\hline \multirow{4}{*}{ 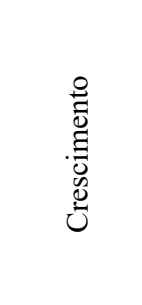 } & O poder decisório de nossa empresa é dividido entre muitos donos e investidores. \\
\hline & $\begin{array}{l}\text { Em nossa organização temos várias especializações (contadores, engenheiros, administradores, etc.) } \\
\text { e, com isso, nos tornamos diferenciados. }\end{array}$ \\
\hline & $\begin{array}{l}\text { O processamento das informações pode ser descrito como monitorador de desempenho e facilitador } \\
\text { de comunicação entre os departamentos. }\end{array}$ \\
\hline & $\begin{array}{l}\text { A maioria das decisões na nossa empresa é tomada por grupos de gestores de nível intermediário } \\
\text { que utilizam alguma sistemática ainda bem superficial. }\end{array}$ \\
\hline \multirow{4}{*}{ 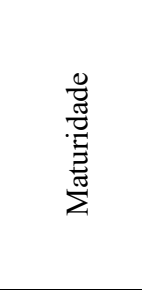 } & $\begin{array}{l}\text { Como empresa, somos maiores do que a maioria dos nossos concorrentes, mas não tão grandes } \\
\text { como deveríamos ser. }\end{array}$ \\
\hline & O poder decisório de nossa empresa está concentrado nas mãos de um número de acionistas. \\
\hline & $\begin{array}{l}\text { A estrutura organizacional da empresa é baseada na visão departamental e funcional (p. ex.: setores } \\
\text { contábil, financeiro, produção, comercial, etc.). }\end{array}$ \\
\hline & $\begin{array}{l}\text { O processamento de informações é sofisticado e necessário para a produção eficiente e para atingir } \\
\text { os resultados planejados. }\end{array}$ \\
\hline \multirow[b]{2}{*}{ 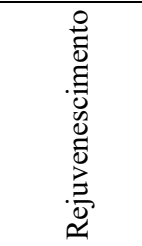 } & Somos uma organização com diretores e acionistas. \\
\hline & $\begin{array}{l}\text { A estrutura organizacional é divisional ou matricial com sofisticado sistema de controle. Divisional } \\
\text { é dividido, p. ex., por setores infantil, masculino, feminino; setor de laticínios, açougue e frutas. } \\
\text { Matricial é formado pelas estruturas funcional + divisional, utilizado por empresas que precisam } \\
\text { acompanhar projetos específicos (p. ex.: construção de uma obra (projeto); contabilidade da obra; } \\
\text { técnico da obra; engenheiro da obra, etc.). }\end{array}$ \\
\hline
\end{tabular}




\begin{tabular}{|c|l|}
\hline \multirow{2}{*}{} & $\begin{array}{l}\text { O processamento de informações é muito completo e utilizado na coordenação de diversas } \\
\text { atividades para melhor servir aos mercados. }\end{array}$ \\
\cline { 2 - 3 } & $\begin{array}{l}\text { A maioria das decisões na nossa empresa é feita por gerentes, forças-tarefas, equipes de projeto, que } \\
\text { estão tentando facilitar o crescimento por meio da participação. }\end{array}$ \\
\hline \multirow{3}{*}{$:$} & A estrutura organizacional da empresa é centralizada, com poucos sistemas de controle. \\
\cline { 2 - 3 } & O processamento de informações não é muito sofisticado. \\
\cline { 2 - 3 } & O processo decisório é centralizado na alta administração e considerado não muito complexo. \\
\cline { 2 - 3 } & A maioria das decisões na nossa empresa é feita por alguns gerentes conservadores. \\
\hline
\end{tabular}

Fonte: Lester, Parnell e Carraher (2003).

Para estabelecer o tamanho da organização, utilizou-se dos critérios de classificação estabelecida pela parceria formada entre SEBRAE (Serviço Brasileiro de Apoio às Micro e Pequena Empresa) e o DIEESE (Departamento Intersindical de Estatística e Estudos Socioeconômicos), que dividem os estabelecimentos em: Microempresa; Pequena empresa; Média empresa e Grande empresa. Sendo, microempresa as empresas que tenham até 19 pessoas; pequena empresa aquelas que possuam de 20 a 99 pessoas; média empresa as que contam com 100 a 499 pessoas; e grande empresa as que apresentem de 500 ou mais pessoas.

Os parâmetros da diversidade de produtos foram extraídos do estudo de Malmi (1999) que estabeleceu 3 escalas: (i) 1-10 produtos; (ii) 11-100 produtos e (iii) acima 100 produtos. O nível de escolaridade foi medido de acordo com o grau de formação do respondente, podendo ser: ( $i$ ) até o ensino médio completo; ( $i i)$ o ensino superior completo; e (iii) a pós-graduação completa.

Dessa forma, por meio da variável dos estágios do ciclo de vida organizacional, pretendeu-se analisar sua influência no uso das medidas de desempenho em empresas industriais do Estado da Paraíba, as demais variáveis foram utilizadas para controlar os possíveis efeitos de fatores diversos aos impactos do CVO.

\subsection{Método de Análise}

Para alcançar os objetivos traçados neste estudo os dados foram analisados por meio dos seguintes modelos de regressão linear multivariada:

$$
\begin{gathered}
\mathrm{MDF}=\beta 0+\beta 1 \mathrm{CVO}+\beta 2 \mathrm{TO}+\beta 3 \mathrm{DP}+\beta 4 \mathrm{ESC}+\mu \\
\mathrm{MDNF}=\beta 0+\beta 1 \mathrm{CVO}+\beta 2 \mathrm{TO}+\beta 3 \mathrm{DP}+\beta 4 \mathrm{ESC}+\mu
\end{gathered}
$$

Onde, MDF consiste na média da intensidade de uso das medidas de desempenho financeiras e MDNF constitui a média da intensidade de uso das medidas de desempenho não financeiras; CVO representa o estágio do ciclo de vida organizacional; TO indica o tamanho da organização; DP expressa à diversidade de produtos; ESC consiste na escolaridade do respondente e $\beta$ representa os parâmetros da regressão, sendo o termo $\beta 0$ o coeficiente linear e os termos $\beta 1, \beta 2, \ldots \beta \mathrm{n}$ os coeficientes angulares.

Para Corrar, Paulo e Dias Filho (2014), a regressão linear múltipla consiste na busca por descrever o comportamento de determinada variável, denominada dependente, com base nos valores de uma ou mais variáveis, denominadas independentes. Antes da análise da regressão, foram realizados testes para verificar os pressupostos que validam a regressão linear múltipla, e dentre esses testes, executaram-se os testes de: correlação de Spearman (multicolinearidade), Shapiro-Francia (normalidade) e Breusch-Pagan (heterocedasticidade).

Para se calcular as médias das medidas de desempenho organizacional, foi disponibilizado, a cada participante, um conjunto de 39 medidas, sendo 25 classificadas como medidas de desempenho financeiras e 14 medidas de desempenho não financeiras. Cada medida de desempenho recebeu uma pontuação que variou de 0 a 7, sendo 0 atribuído no caso de a empresa não utilizar tal medida, 1, no caso de utilizar muito pouco; 7 em casos de muito uso, e as demais numerações, para usos intermediários.

Após a atribuição das notas a cada medida de desempenho, realizou-se a soma que o participante atribuiu a todas as medidas de desempenho financeiras. O resultado foi divido por 25. Procedimento semelhante foi realizado para cada participante, utilizando as medidas de desempenho não financeiras, sendo 
que, para este caso, dividiu o resultado pela quantidade total das medidas não financeiras, que foi 14. Esses resultados constituíram as variáveis: Média das Medidas de Desempenho Financeiras e Média das Medidas de Desempenho não Financeiras.

As variáveis de identificação de cada estágio do ciclo de vida foram submetidas à avaliação de cada empresa, assinalando de acordo com uma Escala do tipo Likert de cinco pontos, dentro de uma escala crescente de concordância as seguintes alternativas: $1=$ Discordo totalmente; $2=$ Discordo parcialmente; $3=$ Nem concordo nem discordo; 4 = Concordo parcialmente; $5=$ Concordo plenamente.

As empresas foram classificadas nos estágios de vida por meio do somatório dos valores individuais de cada assertiva, formando, assim, cinco somas para cada empresa participante e para cada estágio do ciclo de vida organizacional. Em seguida, para classificar a empresa no estágio correspondente, analisou-se, dentre os cinco somatórios, qual correspondia à maior soma. $\mathrm{O}$ estágio que continha a maior soma foi aquele em que a empresa foi classificada. Nos casos em que ocorreram classificações em mais de um estágio para a mesma empresa, utilizou-se, como critério de desempate, o mesmo adotado por Santos, Corrêa e Beuren (2016), que foi a escolha do menor estágio.

Para fins de confirmação da classificação das empresas nos estágios do ciclo de vida organizacional, utilizou-se da análise discriminante, ou seja, mesmo procedimento adotado por Klann et al. (2012), Faveri et al. (2014) e Santos, Corrêa e Beuren (2016). Essa técnica estatística tem como objetivo resolver problemas de classificação e previsão de elementos observados.

Após a classificação das empresas nos estágios do CVO, criaram-se variáveis Dummy, sendo 1 para os casos em que as empresas se encontrassem em determinados estágios do ciclo de vida (nascimento, crescimento, maturidade, rejuvenescimento e declínio) e 0 para os casos de não pertencer a estes estágios. $\mathrm{O}$ objetivo dessa classificação foi estabelecer uma referência para o desenvolviemento da análise da influência dos estágios do ciclo no uso das medidas de desempenho.

\section{Análise e Discussão dos Resultados}

Esta seção apresenta os resultados obtidos com a pesquisa e está dividida em duas partes. A primeira apresenta a análise da influência dos estágios de vida organizacional no uso de medidas de desempenho. Na segunda parte é abordada a análise do teste de hipótese da pesquisa.

\subsection{Análise da influência dos estágios de vida no uso de medidas de desempenho}

A avaliação da influência exercida pelos estágios de ciclo de vida organizacional no uso das medidas de desempenho (financeiras e não financeiras) em empresas industriais da Paraíba foi realizada por meio da análise de regressão linear multivariada. Assim, considerando a importância da significância estatística que é influenciada pelas variáveis utilizadas no modelo de regressão, optou-se por utilizar o método de busca sequencial das variáveis conhecido por backward.

O método de busca sequencial backward estima a variável estatística primeiramente com todas as variáveis independentes e, a partir dele, eliminam variáveis até alcançarem a melhor medida dentro do critério utilizado. Ou seja, partindo da modelagem inicial que contém todas as variáveis explicativas, testam-se os parâmetros e eliminam-se as variáveis que não apresentarem significância estatística (CORRAR; PAULO; DIAS FILHO, 2014; FÁVERO et al., 2009). Dentre as vantagens desse procedimento, está o tratamento de eventuais problemas de multicolinearidade.

Nesta perspectiva, efetuaram-se os testes de regressão linear múltipla de forma separada. Inicialmente foram realizados os testes que tinham como variável dependente as medidas de desempenho financeiras (Equação 1). Em seguida foram realizados os testes com as medidas não financeiras de desempenho (Equação 2). Nos dois testes realizados, foram utilizados um conjunto de 12 variáveis explicativas representadas pelos estágios do ciclo de vida organizacional, tamanho da organização, quantidade de produtos comercializados e escolaridade.

O primeiro teste realizado com a média das medidas financeiras de desempenho como variável dependente resultou em sete modelos, de modo que se eliminava uma variável explicativa a cada modelo, até apresentar apenas as variáveis com parâmetros significantes a 10\%. Assim, do conjunto de 12 variáveis explicativas utilizadas inicialmente, as que apresentaram significância estatística foram: Crescimento, 
Maturidade, Rejuvenescimento e Microempresa.

Em seguida, foi realizado procedimento semelhante, sendo que, desta vez, utilizando como variável dependente a média das medidas não financeiras de desempenho. Como resultado, obteve a previsão de dez modelos estatísticos, até evidenciar apenas as variáveis independentes que possuem significância estatística. Nesse segundo teste, apenas o estágio de vida rejuvenescimento mostrou-se significante para explicar as variações no uso das medidas não financeiras de desempenho.

Após a identificação dos modelos que melhor explicam as variações no uso das medidas de desempenho, a Tabela 1 apresenta os modelos propostos que explicam 21,9\% e 11,8\% da variância do uso das medidas de desempenho financeiras e não financeiras, respectivamente.

Tabela 1 - Resultado da regressão utilizando todas variáveis explicativas

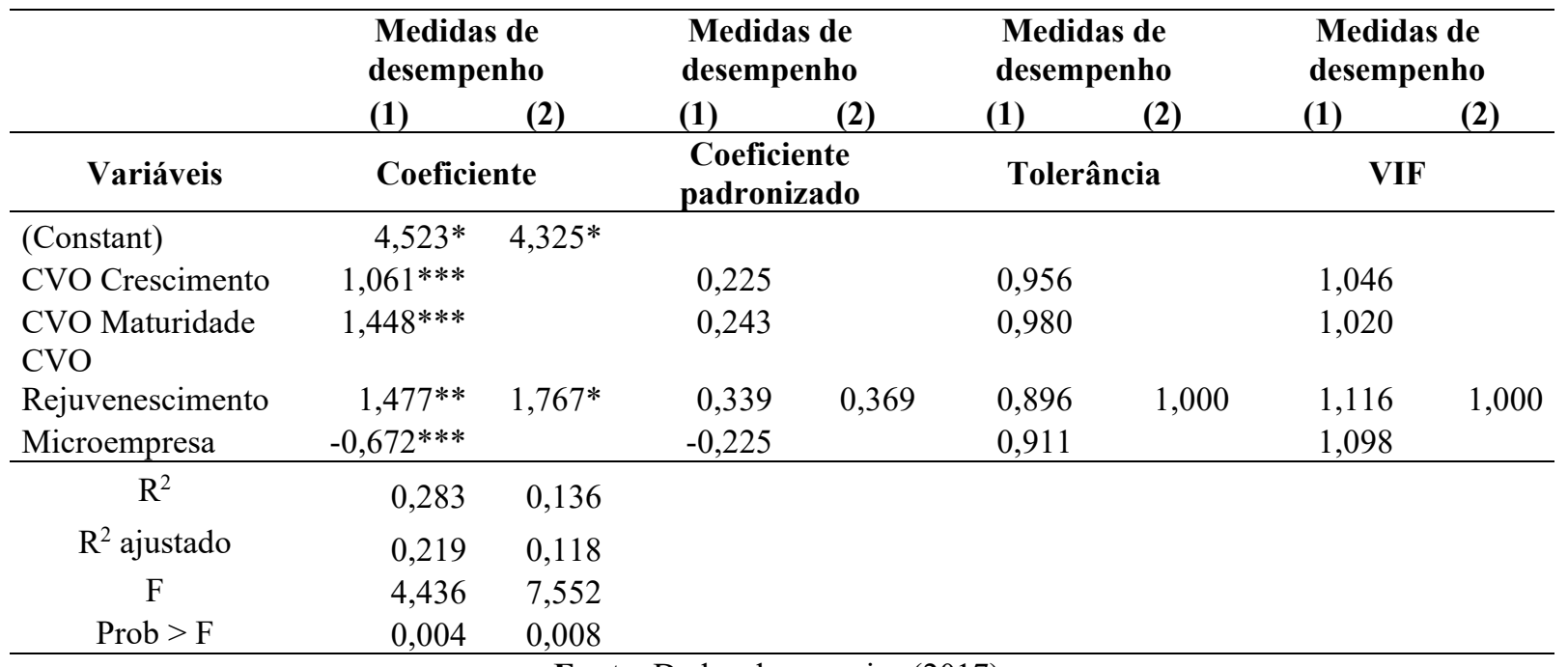

Fonte: Dados da pesquisa (2017).

Obs.: A correlação é significativa *ao nível de $1 \%$; ** ao nível de $5 \%$ e *** ao nível de $10 \%$. Legenda: (1) Financeira; (2) Não Financeira.

Os resultados apresentados na Tabela 1 correspondem aos modelos que evidenciaram apenas variáveis com parâmetros significantes a $10 \%$. Nota-se que os valores de tolerância são altos e que a estatística VIF (variance inflation factor) apresentou valores abaixo de 5.

Para Fávero et al. (2009), a tolerância indica a proporção da variação de uma variável explicativa que independe das outras variáveis explicativas. Logo, valores baixos de tolerância sinalizam possíveis problemas de multicolinearidade. No caso da estatística VIF, Gujarati (2011) afirma que um VIF acima de 10 é indicativo de multicolinearidade. Portanto, nos dois testes apresentados, constata-se que não há problemas de tal natureza.

Assim, as equações (1) e (2) podem ser representada da seguinte forma:

$$
\begin{gathered}
\mathrm{MDF}=4,523+1,061 . \mathrm{CVO} \text { Crescimento }+1,448 . \mathrm{CVO} \text { Maturidade }+1,477 . \mathrm{CVO} \text { Rejuvenescimento } \\
-0,672 . \text { Microempresa }+\mu \\
\operatorname{MDNF}=4,325+1,767 . \mathrm{CVO} \text { Rejuvenescimento }+\mu
\end{gathered}
$$

A partir desses resultados, pode-se observar que o ciclo de vida organizacional exerce influência sobre o uso das medidas de desempenho e que essa influência difere de acordo com o estágio de vida em que se encontram as organizações, sendo a intensidade também diferente quanto ao uso de medidas financeiras e não financeiras.

Logo, constata-se que as medidas financeiras de desempenho sofrem interferências significativas estatisticamente, dos estágios crescimento; maturidade e rejuvenescimento. Já para as medidas de desempenho não financeiras, os resultados apontaram para influência apenas do estágio de rejuvenescimento. 
Conclui-se que, aproximadamente, 22\%, 24\% e 33\% das variações na intensidade de uso das medidas de desempenho financeiras são atribuídas aos estágios de vida de crescimento, maturidade e rejuvenescimento, respectivamente. Destaca-se, também, que o uso das medidas financeiras sofre uma influência negativa de 0,672 para os casos em que a empresa seja classificada como Microempresa.

Isso significa que, caso a empresa esteja classificada como microempresa, haverá uma tendência de usar em menor intensidade as medidas financeiras para acompanhar o desempenho organizacional. Nos casos das medidas não financeiras, os resultados apontaram que, aproximadamente, $37 \%$ das variações na intensidade de uso das medidas são afetadas pelo estágio de ciclo de vida rejuvenescimento.

Outros testes adicionais de regressão foram realizados, sendo que, desta vez, utilizando como variáveis explicativas apenas os estágios do ciclo de vida. Os resultados são apresentados na Tabela 2.

Tabela 2 - Resultado da regressão utilizando como variáveis explicativas apenas os estágios de vida

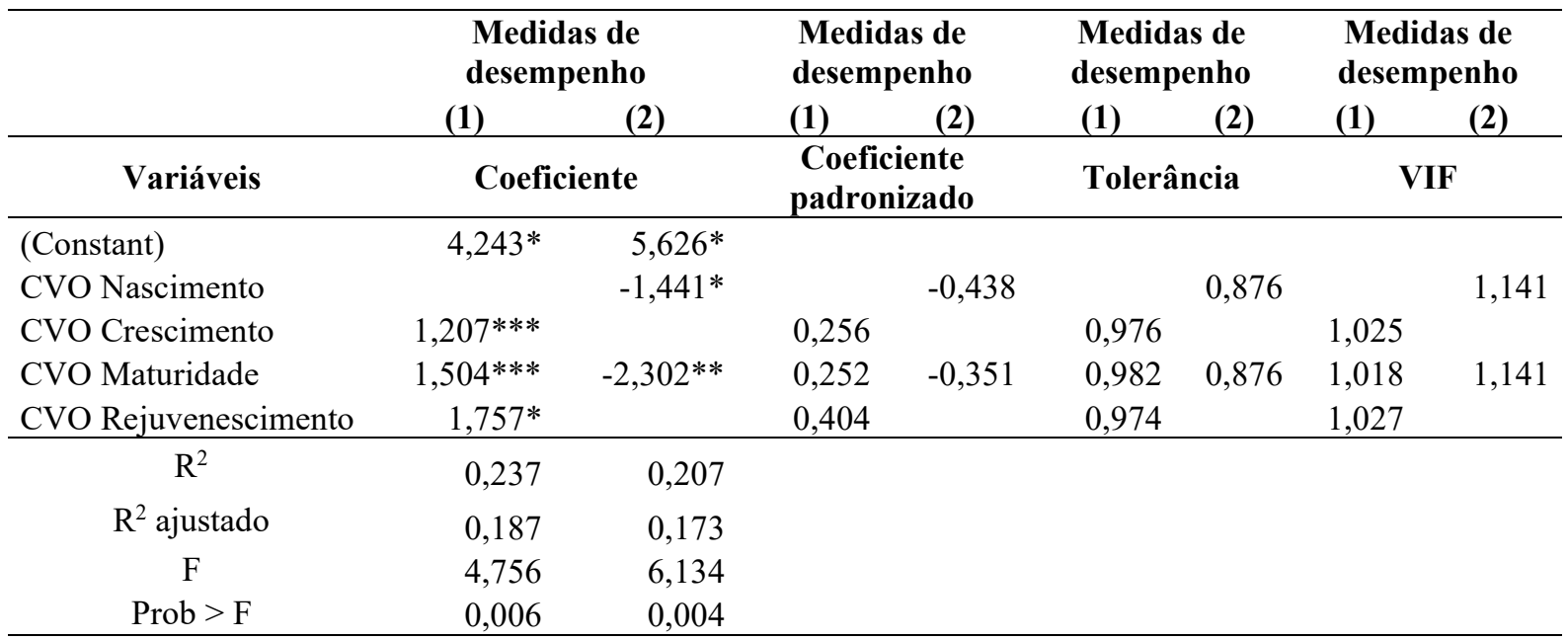

Fonte: Dados da pesquisa (2017).

Obs.: A correlação é significativa *ao nível de $1 \%$; ** ao nível de $5 \%$ e *** ao nível de $10 \%$.

Legenda: (1) Financeira; (2) Não Financeira.

Conforme pode ser observado, os resultados são alterados quando são utilizados apenas os estágios do ciclo de vida organizacional como variáveis independentes para explicarem as variações na intensidade de uso das medidas de desempenho. Com esses resultados, notam-se alterações nos estágio de vida que influenciam a intensidade de uso das medidas não financeiras, ou seja, o nascimento e a maturidade surgem como fatores determinantes da variação no uso de medidas de desempenho não financeiras.

Diante dos resultados obtidos, tem-se que o nascimento e a maturidade são responsáveis por, aproximadamente, $44 \%$ e $35 \%$, respectivamente, da variação na intensidade de uso dessas medidas. No entanto, as variações que são provocadas pelas fases nascimento e maturidade são negativas. Ou seja, caso a empresa se encontre nesses estágios, há uma tendência de que ela use com menos intensidade as medidas de desempenho não financeiras.

Ademais, os estágios de crescimento, maturidade e rejuvenescimento que se mostraram significantes quando da utilização com as demais variáveis na Tabela 1, apresentaram-se como fatores determinantes da variação na intensidade de uso das medidas de desempenho financeiras.

Assim, diante dos resultados apresentados, constatou-se a influência dos estágios de vida no uso das medidas de desempenho.

\subsection{Teste da hipóstese da pesquisa}

As Tabelas 1 e 2 possibilitaram realizar uma avaliação da hipótese levantada nesta pesquisa, sendo possível concluir, por meio dos resultados apresentados, que os estágios do ciclo de vida organizacional exercem influências nas medidas de desempenho. O Quadro 3 apresenta uma síntese da avaliação da hipótese da pesquisa: 
Quadro 3 - Síntese da avaliação da hipótese da pesquisa

\begin{tabular}{|l|l|}
\hline \multicolumn{1}{|c|}{ Hipótese } & Resultado \\
\cline { 2 - 3 } $\begin{array}{l}\text { H0 - Os estágios iniciais (nascimento e crescimento) exercerão influência positiva nas medidas } \\
\text { de desempenho não financeiras maiores do que nas medidas financeiras, quando comparados } \\
\text { com os demais estágios. }\end{array}$ & Rejeitada \\
\hline \multicolumn{2}{|c|}{ Fonte: Elaboração própria, 2017. } \\
\hline
\end{tabular}

No que se referem à influência exercida pelos estágios de vida nas medidas de desempenho, as Tabelas 1 e 2 apresentaram que a fase crescimento exerce uma menor influência no uso das medidas financeiras quando comparada com as fases maturidade e rejuvenescimento. No que diz respeito ao uso das medidas não financeiras, os estágios de nascimento e maturidade apresentaram influências negativas.

Então, os resultados encontrados nesta pesquisa apontam que os estágios mais avançados exercem uma influência na intensidade do uso das medidas de desempenho maior do que os estágios iniciais. Esses resultados refutam os resultados de Larimo, Nguyen e Ali (2016) que afirmam as empresas têm certas preferências por usar medidas não financeiras em estágios de formação, ou seja, nos estágios iniciais.

\section{Considerações Finais}

Este estudo teve como objetivo central analisar a influência do ciclo de vida organizacional no uso de medidas de desempenho. Para tanto, fez-se elucidar o seguinte problema de pesquisa: qual a influência dos estágios do ciclo de vida organizacional no uso de medidas de desempenho (financeira e não financeira) em empresas industriais da Paraíba?

Primeiramente, identificaram-se os estágios de vida das empresas participantes da pesquisa. Concluiu-se que as organizações mudam de estágios de vida, na medida em que são alteradas as configurações do contexto empresarial, estratégia e estrutura organizacional. Já que se identificaram empresas nos cinco estágios de vida do modelo de CVO de Lester, Parnell e Carraher (2003). Esse resultado corrobora que as organizações passam por diversas fases de vida à medida que crescem e se desenvolvem, formando assim um ciclo de vida organizacional (HANKS et al., 1993; MILLER; FRIESEN, 1984; MINTZBERG, 1984).

Quanto ao uso das medidas de desempenho, concluiu-se que as empresas participantes desta pesquisa utilizam tanto medidas financeiras, quanto medidas não financeiras para acompanhar o desempenho organizacional, sendo que, em média, usam-se as medidas financeiras de uma forma mais intensa. Com isso, nota-se possíveis relações com os estudos de Miranda et al. (2003), Lopes e Beuren (2015) e Vargas et al. (2016) que ao se referirem as medidas de desempenho afirmaram que as organizações se utilizam tanto de métricas financeiras, quanto não financeira. Podendo, inclusive, se utilizar de combinações de medidas financeiras e não financeiras.

Verificou-se a influência que os estágios do ciclo de vida exercem sobre as medidas de desempenho e qual a intensidade dessa influência. Os resultados possibilitaram inferir que os estágios de crescimento, maturidade e rejuvenescimento e o fato de a empresa ser microempresa exercem influências significantes nas intensidades de uso das medidas financeiras de desempenho.

E no tocante às medidas não financeiras, as variáveis que se mostraram influentes foram nascimento e rejuvenescimento. No entanto, a influência do estágio nascimento foi negativa. Ou seja, à medida que a organização apresenta características da fase nascimento, menor será a intensidade de uso das medidas não financeiras.

Esses resultados ratificam e fortalecem que o contexto empresarial, traduzido por meio da teoria do ciclo de vida organizacional, é um dos fatores determinantes que interferem na escolha de quais medidas de desempenho devem ser adotadas para avaliar o desempenho organizacional (YAN; GRAY, 1995; LARIMO; NGUYEN; ALI, 2016). 
Portanto, acredita-se que este trabalho tem contribuições teóricas e práticas. Com relação aos aspectos teóricos, esta pesquisa fornece informações que enriquece a literatura no que diz respeito ao entendimento do uso dos controles gerenciais, sobretudo, da compreensão da influência dos estágios do ciclo de vida no uso de medidas de desempenho.

Quanto aos aspectos práticos, esta pesquisa proporciona aos gestores o conhecimento de que as organizações passam por diferentes estágios de vida e que à medida que as empresas mudam de estágios, é necessário realizar adaptações nos controles gerenciais para enfrentar possíveis problemas que surjam em decorrência do processo de crescimento.

No que se refere à limitação da pesquisa, este estudo compreendeu apenas uma pequena parcela das empresas industriais do Estado da Paraíba que foi constituída a partir do retorno obtido por meio dos questionários enviados. Sendo assim, os resultados têm validade apenas dentro de um contexto específico, que é sua amostra, não podendo ser estendido às demais empresas industriais paraibanas que não participaram da pesquisa, em virtude da impossibilidade de generalizações, embora tenha se utilizado o método hipotético dedutivo para formular a hipótese de pesquisa.

Outra limitação deste estudo diz respeito ao procedimento de coleta de dados. Como as técnicas e os instrumentos de observação consistiram na observação indireta, por meio do uso de questionários, os resultados apresentam apenas a percepção dos gestores quanto ao uso dos instrumentos gerenciais e das características empresariais elencados nos questionários. Sugere-se o uso de outras técnicas de pesquisa, como o uso da observação direta e estudos de casos.

Por fim, o setor econômico ao qual a empresa industrial pertence não foi uma variável considerada neste estudo. Embora o setor econômico não seja uma variável conhecida que influencia na relação da estratégia do ciclo de vida, é possível que diferentes setores adotem diferentes medidas de desempenho organizacional, de tal forma que possa avaliar melhor as atividades de um setor específico. Importante destacar que essa última limitação já estava presente no estudo de Lester, Parnell e Carraher (2003).

\section{REFERÊNCIAS}

ADIZES, I. Os ciclos de vida das organizações: como e por que as empresas crescem e morrem e o que fazer a respeito. São Paulo: Pioneira, 1990.

ALMEIDA, I. X. D.; WERNKE, R. Estilos Gerenciais dos Dirigentes de Pequenas Empresas: Estudo Baseado no Ciclo de Vida Organizacional. Revista de Empreendedorismo e Gestão de Pequenas Empresas, São Paulo, v. 7, n. 3, p. 110-140, 2018.

AUZAIR, S. M.; LANGFIELD-SMITH, K. The effect of service process type, business strategy and life cycle stage on bureaucratic MCS in service organizations. Management Accounting Research, v. 16, n. 4, p. 399 $421,2005$.

BEUREN, I. M.; RENGEL, S.; HEIN, N. Ciclo de vida organizacional no modelo de Lester, Parnell e Carraher (2003) e na Lógica Fuzzy: Classificação de Empresas de um Segmento Industrial de Santa Catarina. Revista de Administração, São Paulo, v. 47, n. 2, p. 197-216, 2012.

BEUREN, I. M.; RENGEL, S.; RODRIGUES JUNIOR, M. M. Relación de los atributos de contabilidad gerencial con las fases del ciclo de vida organizacional. Innovar, Bogotá, v. 25, n. 57, p. 63-78, 2015.

BOND, E. Medição de desempenho para gestão da produção em um cenário de cadeia de suprimentos. 2002. 136 f. Dissertação (Mestrado em Engenharia de Produção) - Programa de Pós-Graduação em Engenharia de Produção, Escola de Engenharia de São Carlos da Universidade de São Paulo, São Paulo, 2002.

BORINELLI, M. L. A identificação do ciclo de vida das pequenas empresas através das demonstrações contábeis. 1998. 179 f. Dissertação (Mestrado em Engenharia de Produção) - Programa de Pós-Graduação em Engenharia de Produção. Universidade Federal de Santa Catarina, Florianópolis, 1998.

CALlADO, A. A. C. Relações entre o número de indicadores de desempenho utilizados e fatores contingenciais: evidências do contexto de uma cadeia agroalimentar de suprimentos. Contabilidade Vista $\&$ Revista, Belo Horizonte, v. 27, n. 2, p. 48-63, 2016. 
CAllado, A. A. C.; CAllado, A. L. C.; WEGNER, D. Estudo Comparativo entre Organizações Agroindustriais sobre adoção de Indicadores de Desempenho de Cadeia de Suprimento e Características de Estratégia. In: CONGRESSO BRASILEIRO DE CUSTOS, 22., 2015, Foz do Iguaçu. Anais eletrônicos... Foz do Iguaçu: ABC, 2015. Disponível em: <https://anaiscbc.emnuvens.com.br/anais/article/view/4002>. Acesso em: 25 jan. 2016.

CAllado, A. L. C.; CAllado, A. A. C.; AlMEIDA, M. A. A. utilização de indicadores gerenciais de desempenho industrial no âmbito de agroindústrias. Revista Eletrônica Sistema \& Gestão, Niterói, v. 2, n. 2, p. $102-118,2007$.

CHAVES, L. D.; ALCÂNTARA, L. C.; ASSUMPÇAO, R. P. Medidas de Desempenho na Logística Reversa: o caso de uma empresa do setor de bebidas. Relatórios de Pesquisa em Engenharia de Produção da UFF, Rio de Janeiro, v. 8, n.2, 2008.

CHURCHILL, N. C.; LEWIS, V. L. The five stages of small business growth. Harvard Business Review, v. 61, n. 3 , p. $30-50,1983$.

CORRAR, L. J.; PAULO, E.; DIAS FILHO, M. Análise multivariada: para os cursos de administração, ciências contábeis e economia. 1' . ed. - 7. reimpr. São Paulo: Atlas, 2014.

CORREIA, R. B. et al. Análise dos modelos de ciclo de vida organizacional aplicados em pesquisas empíricas. In: CONGRESSO BRASILEIRO DE CUSTOS, 17., 2010, Belo Horizonte. Anais eletrônicos... Belo Horizonte: ABC, 2010. Disponível em: <https://anaiscbc.emnuvens.com.br/anais/article/view/780>. Acesso em: 15 jan. 2016.

CUNHA, P. R. da; KLANN, R. C.; LAVARDA, C. E. F. Ciclo de vida organizacional e controle gerencial: uma análise dos artigos em periódicos internacionais de contabilidade. Revista de Gestão, Finanças e Contabilidade, Senhor do Bonfim, v. 3, n. 3, p. 170-186, 2013.

FAVERI, D. B. de et al. Relação do Ciclo de Vida Organizacional com o Planejamento: um Estudo com Empresas Prestadoras de Serviços Contábeis do Estado de Santa Catarina. Revista de Educação e Pesquisa em Contabilidade, Brasília, v. 8, n. 4, p. 382-402, 2014.

FÁVERO, L. P. et al. Análise de dados: modelagem multivariada para tomada de decisões. Rio de Janeiro: Elsevier, 2009.

FEDERAÇÃO DAS INDÚSTRIAS DO ESTADO DA PARAÍBA (FIEP). Cadastro Industrial Paraíba. Paraíba. 2015. Disponivel em: <http://www.fiepb.com.br/industria/empresas/avancado>. Acesso em: 15 dez. 2015.

FLAMHOLTZ, E. Managing the Transition from an Entrepreneurship to a Professionally Managed Firm. San Francisco, CA: Jossey-Bass, 1986.

FREZATTI, F. et al. Perfil de planejamento e ciclo de vida organizacional nas empresas brasileiras. Revista de Administração, São Paulo, v. 45, n. 4, p. 383-399, 2010.

FREZATTI, F. et al. Estágios do Ciclo de Vida e Perfil de Empresas Familiares Brasileiras. Revista de Administração de Empresas, São Paulo, v. 57, n. 6, nov./dez. 2017.

GARENGO, P.; NUDURUPATI, S.; BITITCI, U. Understanding the relationship between PMS and MIS in SMEs: An organizational life cycle perspective. Computers in Industry, v. 58, n. 7, p. 677-686, 2007.

GERINGER, J. M.; HEBERT, L. Measuring performance of international joint ventures. Journal of International Business Studies, v. 22, n. 2, p. 249-263, 1991.

GIL, A. C. Métodos e técnicas de pesquisa social. 6 ${ }^{\text {a }}$ 3. reimpr. ed. São Paulo: Atlas, 2010.

GRANLUND, M.; TAIPALEENMÄKI, J. Management control and controllership in new economy firms-a life cycle perspective. Management Accounting Research, v. 16, n. 1, p. 21-57, 2005.

GREINER, L. E. Evolution and revolution as organizations grow. Harvard Business Review, v. 50, n. 1, p. $37-46,1972$. 
GUJARATI, D. N. Econometria Básica. 5a. ed. Porto Alegre: Bookman, 2011.

HANKS, S. H. et al. Tightening the life cycle construct: A taxonomic study of growth stage configurations in high-technology organizations. Entrepreneurship: Theory and Practice, v. 18, n. 2, p. 5-30, 1993.

KALLUNKI, J. P.; SILVOLA, H. The effect of organizational life cycle stage on the use of activity-based costing. Management Accounting Research, v. 19, n. 1, p. 62-79, 2008.

KLANN, R. C. et al. Relação entre o ciclo de vida organizacional e o planejamento em empresas metalúrgicas do município de Brusque-SC. Revista de Contabilidade e Organizações, Ribeirão Preto, v. 6, n. 16, p. 119$142,2012$.

KONSTA, K.; PLORIMATOU, E. Key performance indicators (KPIs) and shipping companies performance evaluation: the case of greek tanker shipping companies. International Journal of Business and Management, v. 7, n. 10, p. 142-155, 2012.

LARIMO, J.; NGUYEN, H. L.; ALI, T. Performance measurement choices in international joint ventures:What factors drive them? Journal of Business Research, v. 69, n. 2, p. 877-887, 2016.

LESTER, D. L.; PARNELL, J. A.; CARRAHER, S. Organizational life cycle: a five stage empirical scale. The International Journal of Organizational Analysis, v. 11, n. 4, p. 339-354, 2003.

LOPES, I. F.; BEUREN, I. M. Comportamento dos custos e sua relação com medidas não financeiras de desempenho em companhias aéreas. In: CONGRESSO BRASILEIRO DE CUSTOS, 22., 2015, Foz do Iguaçu. Anais eletrônicos... Foz do Iguaçu: ABC, 2015. Disponível em: $<$ https://anaiscbc.emnuvens.com.br/anais/article/view/3990>. Acesso em: 10 jan. 2016.

MALMI, T. Activity-based costing diffusion across organizations: an exploratory empirical analysis of Finnish firms. Accounting, organizations and society, v. 24, n. 8, p. 649-672, 1999.

MARCONI, M. D. A.; LAKATOS, E. M. Técnicas de pesquisa: planejamento e execução de pesquisas, amostragens e técnicas de pesquisa, elaboração, análise e interpretação de dados. $7^{\mathrm{a}}$. ed. São Paulo: Atlas, 2015 .

MILLER, D.; FRIESEN, P. H. A longitudinal study of the corporate life cycle. Management science, v. 30 , n. 10, p. $1161-1183,1984$.

MINTZBERG, H. Power and organization life cycles. Academy of Management Review, v. 9, n. 2, p. $207-$ 224,1984

MIRANDA, L. C. et al. Indicadores de Desempenho Empresarial divulgados por empresas Norte Americanas. Contabilidade Vista e Revista, Belo Horizonte, v. 14, n. 2, p. 85-103, 2003.

MIRANDA, L. C.; SILVA, J. D. G. Medição de Desempenho. In: SCHMIDT, Paulo (Org.). Controladoria: Agregando Valor para a Empresa. Porto Alegre: Bookman, 2002.

MOORES, K.; YUEN, S. Management accounting systems and organizational configuration: a life-cycle perspective. Accounting, Organizations and Society, v. 26, n. 4, p. 351-389, 2001.

NECYK, G. A.; FREZATTI, F. A Contabilidade Gerencial: Uma Perspectiva de Ciclo de Vida de seu Desenvolvimento nas Organizações. Organizações \& Sociedade, Salvador, v. 17, n. 55, p. 725-744, 2010.

NEELY, A. Avaliação do desempenho das empresas. Porquê, o quê e como. Lisboa: Caminho, 2002.

NEELY, A.; GREGORY, M.; PLATTS, K. Performance measurement system design: A literature review and research agenda. International Journal of Operations and Production Management, v. 25, n. 12, p. 1228$1263,2005$.

QUINN, R. E.; CAMERON, K. Organizational life cycles and shifting criterias of effectiveness: some preliminary evidence. Management Science, v. 29, n. 1, p. 33-51, 1983.

RIBEIRO, M. G. C.; MACEDO, M. A. S.; MARQUES, J. A. V. C. Análise da relevância de indicadores financeiros e não financeiros na avaliação de desempenho organizacional: Um estudo exploratório no setor 
brasileiro de distribuição de energia elétrica. Revista de Contabilidade e Organizações, Ribeirão Preto, v. 6, n. 15 , p. $60-79,2012$.

RICHARDSON, J. Pesquisa Social: métodos e técnicas. 3ª ed. São Paulo: Atlas, 1999.

SANTOS, V. D.; CORRÊA, N. L.; BEUREN, I. M. Relação entre ciclo de vida organizacional e uso de instrumentos de Contabilidade Gerencial. In: CONGRESSO BRASILEIRO DE CUSTOS, 23., 2016, Porto de Galinhas. Anais eletrônicos... Porto de Galinhas: ABC, 2016. Disponível em: $<$ https://anaiscbc.emnuvens.com.br/anais/article/view/4098>. Acesso em: 10 dez. 2016.

SCOTT, M.; BRUCE, R. Five stages of growth in small business. Long range planning, v. 20, n. 3, p. 45-52, 1987.

SILVA, M. Z. D.; MARQUES, L.; CECON, B. Relação entre os artefatos de contabilidade gerencial e o ciclo de vida organizacional de empresas do setor de consumo cíclico. Innovar, Bogotá, v. 30, n. 76, p. 105-118, 2020

SIMONS, R. Levers of control. Cambridge, MA: Harvard Business School Press, 1995.

SOUZA, B. C. de; FREZATTI, F.; NECYK, G. A. Ciclo de Vida das Organizações e a Contabilidade Gerencial. Revista Enfoque: Reflexão Contábil, Maringá, v. 27, n. 1, p. 9-22, 2008.

VARGAS, S. B. D. et al. Indicadores Não-Financeiros de Avaliação de Desempenho: Análise de Conteúdo em Relatórios de Administração de Empresas de Telecomunicações. Sociedade, Contabilidade e Gestão, Rio de Janeiro, v. 11, n. 1, 2016.

YAN, A.; GRAY, B. Re-conceptualizing the determinants and measurement of joint. Advances in Global High-TechnologyManagement, v. 5, n. 1, p. 87-113, 1995. 